\title{
Pre-Steady-State Kinetic Analysis of the Reactions of Alternate Substrates with Dialkylglycine Decarboxylase ${ }^{\dagger}$
}

\author{
Shaoxian Sun, Carey K. Bagdassarian, ${ }^{\ddagger}$ and Michael D. Toney* \\ Department of Biochemistry, Albert Einstein College of Medicine, 1300 Morris Park Avenue, Bronx, New York 10461
}

Received August 19, 1997; Revised Manuscript Received December 18, 1997

\begin{abstract}
The pre-steady-state kinetics of the half-reactions of several substrates with dialkylglycine decarboxylase are examined by multiwavelength kinetics and global analysis. The substrates examined fall into two groups: those that exhibit simple, monophasic kinetics and those that exhibit biphasic kinetics. The rate of the AIB half-reaction is likely limited by the decarboxylation step based on the simple kinetics and spectra obtained from global analysis. The spectra for the first species in the transamination halfreactions of L-alanine and L-aminobutyrate show long-wavelength absorption characteristic of a carbanionic quinonoid intermediate. This demonstrates that formation of the external aldimine intermediates and abstraction of the $\mathrm{C}^{\alpha}$ protons from them are rapid. The reactions of the slower substrates L-phenylglycine and 1-aminocyclohexane-1-carboxylate may have external aldimine formation as the rate-determining step. The biphasic reactions of 2-methyl-2-aminomalonate, 1-aminocyclopentane-1-carboxylate, isopropylamine, and glycine all have external aldimine formation as the rapid observable step, based on the spectral changes observed in absorption and circular dichroism measurements. 2-Methyl-2-aminomalonate reacts $\sim 10^{4}$-fold slower than does AIB with dialkylglycine decarboxylase, compared to $\sim 10^{5}$-fold faster with coenzyme in solution. It is proposed that this radical reactivity reversal is due to a slow protein conformational change that is a prerequisite to decarboxylation of MAM, which occurs rapidly thereafter. Circular dichroism measurements on active site bound coenzyme provide evidence supporting this proposal. The binding of the noncovalent inhibitors pyruvate or lactate or the covalently binding inhibitor 1-aminocyclopropane-1-carboxylate all induce a slow change in coenzyme circular dichroism that quantitatively parallels the slow decarboxylation of 2-methyl-2-aminomalonate. Fast circular dichroism changes are seen in the mixing time of these measurements for both 1-aminocyclopropane-1-carboxylate and 2-methyl-2-aminomalonate, indicating rapid external aldimine formation on this longer time scale.
\end{abstract}

The pyridoxal phosphate (PLP) ${ }^{1}$-dependent enzyme dialkylglycine decarboxylase is a unique PLP-dependent enzyme that catalyzes both decarboxylation and transamination in its normal catalytic cycle. The steady-state kinetics of the reactions of alternate substrates with DGD were examined in the previous paper in this issue. That study validated an active site model in which one binding subsite is the locus of all bond making and breaking in the decarboxylation and transamination half-reactions. The unexpectedly low reactivity of several of these substrates prompted the present study in which their pre-steady-state kinetics are analyzed to determine the nature of the rate-determining steps of the reactions.

The substrate analogue MAM is particularly interesting in that it reacts $\sim 10^{4}$-fold slower than does AIB with DGD,

\footnotetext{
Supported by Grant GM54779 from the National Institutes of Health.

* Corresponding author: (e-mail) toney@aecom.yu.edu; (tel) 718430-2347; (fax) 718-430-8565.

Present address: Department of Chemistry, College of William and Mary, Williamsburg, VA 23185.

${ }^{1}$ Abbreviations: DGD, dialkylglycine decarboxylase; PLP, pyridoxal phosphate; PMP, pyridoxamine phosphate; DGD-PLP, PLP form of DGD; DGD-PMP, PMP form of DGD; AIB, $\alpha$-aminoisobutyrate; AC3C, 1-aminocyclopropane-1-carboxylate; AC5C, 1-aminocyclopentane-1-carboxylate; AC6C, 1-aminocyclohexane-1-carboxylate; LDH, lactate dehydrogenase; MAM, 2-methyl-2-aminomalonate; TEA-HCl, triethanolamine hydrochloride.
}

yet it reacts $\sim 10^{5}$-fold faster with free coenzyme in solution. This dramatic reversal in reactivity on going from solution to the DGD active site is difficult to explain on purely mechanistic grounds. Here, evidence is presented that suggests a slow, rate-determining protein conformational change is a prerequisite to decarboxylation of MAM, providing an explanation for the enormous reactivity reversal.

The reactions of several substrates including MAM and AC5C exhibit complex kinetics. Analytical expressions for observed rate constants are derived from schemes describing their reactions. These equations are used in curve-fitting procedures to obtain estimates for the microscopic rate constants for the minimal mechanisms.

\section{EXPERIMENTAL PROCEDURES}

Materials. L-Alanine and AIB were obtained from Sigma and further purified by recrystallization. PLP, NADH, NADPH, pyruvic acid, L-aminobutyrate, L-norvaline, Lnorleucine, glycine, isopropylamine, AC3C, AC5C, AC6C, L-phenylglycine, lactate dehydrogenase, and secondary alcohol dehydrogenase were purchased from Sigma. Lactic acid was from Aldrich. Deuterium oxide $(99.9 \%$ D) was from Cambridge Isotope Laboratories. MAM was synthesized according to the procedures of Bailey et al. (I) and stored at $-20^{\circ} \mathrm{C}$. Stock solutions of MAM were neutralized. 
The detailed synthetic procedure is described in the previous paper in this issue, as are the construction of the DGD expression vector, and enzyme growth and purification. DGD concentration was determined with the Bio-Rad DC (modified Lowry) protein assay, using BSA as the standard.

Rapid-Scanning Stopped-Flow Kinetic Studies. PLP, PMP, and various covalent substrate-coenzyme intermediates have characteristic UV/vis absorption bands above 300 $\mathrm{nm}$ that can be followed spectrophotometrically (2). DGDPLP was rapidly mixed with various substrates in an Applied Photophysics SX.17MV stopped-flow spectrophotometer, and coenzyme absorbance changes in the oxidative decarboxylation or transamination reactions were monitored. Absorbance scans (500) from 300 to $550 \mathrm{~nm}$ were collected on a logarithmic time scale with a UV-enhanced diode array detector (2.6-ms scan time) and were analyzed globally using either SPECFIT (Spectrum Software Associates) or GLINT (Applied Photophysics). Observed rate constants were obtained by fitting the data to either a one- or two-step, irreversible serial first-order mechanism. All reactions were performed under pseudo-first-order conditions with substrates in excess over enzyme. Experimental conditions: $30 \mathrm{mM}$ TEA-HCl pH 8.0, 15-21 $\mu \mathrm{M}$ DGD-PLP, $0.1 \mathrm{M} \mathrm{KCl}$, various concentrations of substrate, $25^{\circ} \mathrm{C}$.

LDH Coupled Assay for Transamination of L-Aminobutyrate. The transamination reaction of $\mathrm{L}$-aminobutyrate with DGD-PLP was coupled to the LDH-catalyzed oxidation of NADH. Absorbance at $340 \mathrm{~nm}$ was followed in the stoppedflow spectrophotometer. Experimental conditions: $30 \mathrm{mM}$ TEA-HCl pH 8.0, 15-21 $\mu \mathrm{M}$ DGD-PLP, $0.1 \mathrm{M} \mathrm{KCl,} 0.25$ M L-aminobutyrate, 1000 units/mL LDH, $0.2 \mathrm{mM}$ NADH; $25{ }^{\circ} \mathrm{C}$. Separate experiments (X. Zhou and M. D. Toney, submitted) have shown this amount of LDH to be sufficient for complete coupling.

Fluorescence Detection of Transamination of L-Aminobutyrate. Steady-state fluorescence spectra of DGD-PLP and DGD-PMP show that the latter form has $\sim 3$-fold greater emission intensity at $390-400 \mathrm{~nm}$ when excited at $335 \mathrm{~nm}$. No fluorescence emission above $\sim 450 \mathrm{~nm}$ was detected for either enzyme form. The transamination reaction of DGDPLP with L-aminobutyrate was followed in the stopped-flow spectrophotometer with monochromated 335-nm excitation and a 360-nm high-pass cutoff filter for emission selection. Experimental conditions: $30 \mathrm{mM}$ TEA-HCl pH 8.0, $21 \mu \mathrm{M}$ DGD-PLP, $0.1 \mathrm{M} \mathrm{KCl}, 0.25 \mathrm{M}$ L-aminobutyrate, $25{ }^{\circ} \mathrm{C}$.

Inhibition by Lactate. The affinity of lactate for DGDPLP was determined by steady-state inhibition studies. The rate of the AIB/pyruvate reaction in the presence of lactate was measured at various concentrations of AIB with several fixed concentrations of lactate and saturating pyruvate. The reaction was coupled to a secondary alcohol dehydrogenasecatalyzed NADPH oxidation (3). The value of $K_{\mathrm{i}}$ was determined by nonlinear curve fitting (GraFit; Erithacus Software) to the Michaelis-Menten equation with a competitive inhibition term.

Circular Dichroism Spectra. CD spectra were obtained on a Jasco J-720 spectrometer at room temperature $(\sim 23$ $\left.{ }^{\circ} \mathrm{C}\right)$. The free enzyme spectrum $(\sim 5 \mathrm{mg} / \mathrm{mL}$ DGD-PLP in $30 \mathrm{mM}$ TEA-HCl, $0.1 \mathrm{M} \mathrm{KCl} ; 70-\mu \mathrm{L}$ microvolume cell) was recorded at $200 \mathrm{~nm} / \mathrm{min}$ from 300 to $500 \mathrm{~nm}$. Spectra of DGD-PLP with pyruvate, lactate, AC3C, AIB, or MAM were obtained as follows. The reactant was mixed with DGD-
PLP, and the resulting solution was replaced in the cell and scanned (2-6-min delay from mixing to scan initiation) repetitively $(1 \mathrm{~min} / \mathrm{scan})$ for $15-25 \mathrm{~min}$. The final DGDPLP concentration was $\sim 5 \mathrm{mg} / \mathrm{mL}$ in all the measurements. The final concentrations of the reactants were as follows: pyruvate, $0.1 \mathrm{M}\left(10 \times K_{\mathrm{i}}\right)$; lactate, $0.54 \mathrm{M}\left(4 \times K_{\mathrm{i}}\right)$; AC3C, $20 \mathrm{mM}\left(14 \times K_{\mathrm{i}}\right)$; AIB, $50 \mathrm{mM}\left(10 \times K_{\mathrm{M}}\right) ; \mathrm{MAM}, 20 \mathrm{mM}$ $\left(3 \times K_{\mathrm{M}}\right)$.

Solvent Isotope Effects. Buffer, substrates, and cofactors were prepared directly in $\mathrm{D}_{2} \mathrm{O}$. A small amount of protium was introduced into the reactions from the enzyme stock solution. The final deuterium content was $\sim 98 \%$. Solvent isotope effects were calculated by directly comparing the kinetic parameters measured in $\mathrm{H}_{2} \mathrm{O}$ and $\mathrm{D}_{2} \mathrm{O}$, ignoring the small amount of protium in the $\mathrm{D}_{2} \mathrm{O}$ reactions.

\section{RESULTS}

Monophasic Pre-Steady-State Kinetics. A rapid change in absorbance followed by a slower one was observed in the AIB decarboxylation half-reaction (data not shown). The time course of the reaction is well fit by a two-step, serial first-order mechanism. The rate constant for the fast phase, $k_{\text {fast }}$, shows a hyperbolic dependence on AIB concentration in the range of $1-20 \mathrm{mM}$. The rate constant for the slow phase is independent of AIB concentration in this range.

The $k_{\text {fast }}$ vs AIB concentration data were fitted to eq 1 . This yielded $k_{\max }=25 \pm 1 \mathrm{~s}^{-1}$ and $K_{\text {app }}=4.9 \pm 0.4 \mathrm{mM}$.

$$
k_{\text {fast }}=\frac{k_{\text {max }} \mathrm{S}}{K_{\text {app }}+\mathrm{S}}
$$

Global analysis (4) of the absorbance changes at all wavelengths collected $(300-550 \mathrm{~nm})$ yields absorption spectra for the initial, intermediate, and final species for the two-step, serial first-order mechanism. The spectra of the first two species (i.e., reaction of the fast enzyme) in the AIB reaction are shown in Figure 1A. The loss in $\sim 400$ $\mathrm{nm}$ absorbance and increase in $\sim 330-\mathrm{nm}$ absorbance is expected for the conversion of DGD-PLP to DGD-PMP. The spectrum of the initial species (A) is similar to that of free DGD-PLP except that the absorption maximum of the longwavelength peak is slightly red-shifted from the free enzyme under similar conditions, indicating that a fast process is complete within the dead time $(\sim 1.5 \mathrm{~ms})$ of the stopped flow. A single isosbestic point at $\sim 352 \mathrm{~nm}$ is observed in the spectral data covering both kinetic phases.

L-Aminobutyrate also reacts in two phases with DGDPLP. The absorption spectra obtained with L-aminobutyrate in the transamination reaction with the fast enzyme (Figure 1B) are representative of the reactions of DGD-PLP with L-amino acids. The spectra are very similar to those obtained with AIB except that transient absorbance at $\sim 500 \mathrm{~nm}$ on the initial species is observed. This is indicative of the buildup of a quinonoid intermediate (5). At saturating concentrations of L-aminobutyrate $\left(0.25 \mathrm{M}, 20 \times K_{\mathrm{M}}\right)$, global analysis of the absorbance changes from 300 to $550 \mathrm{~nm}$ gives $k_{\max }=3.4 \pm 0.2 \mathrm{~s}^{-1}$ for the fast phase, similar to the value $\left(\sim 7 \mathrm{~s}^{-1}\right)$ measured in the pre-steady-state LDH-coupled experiment (see below) and by fluoresence detection of DGD-PMP formation. 

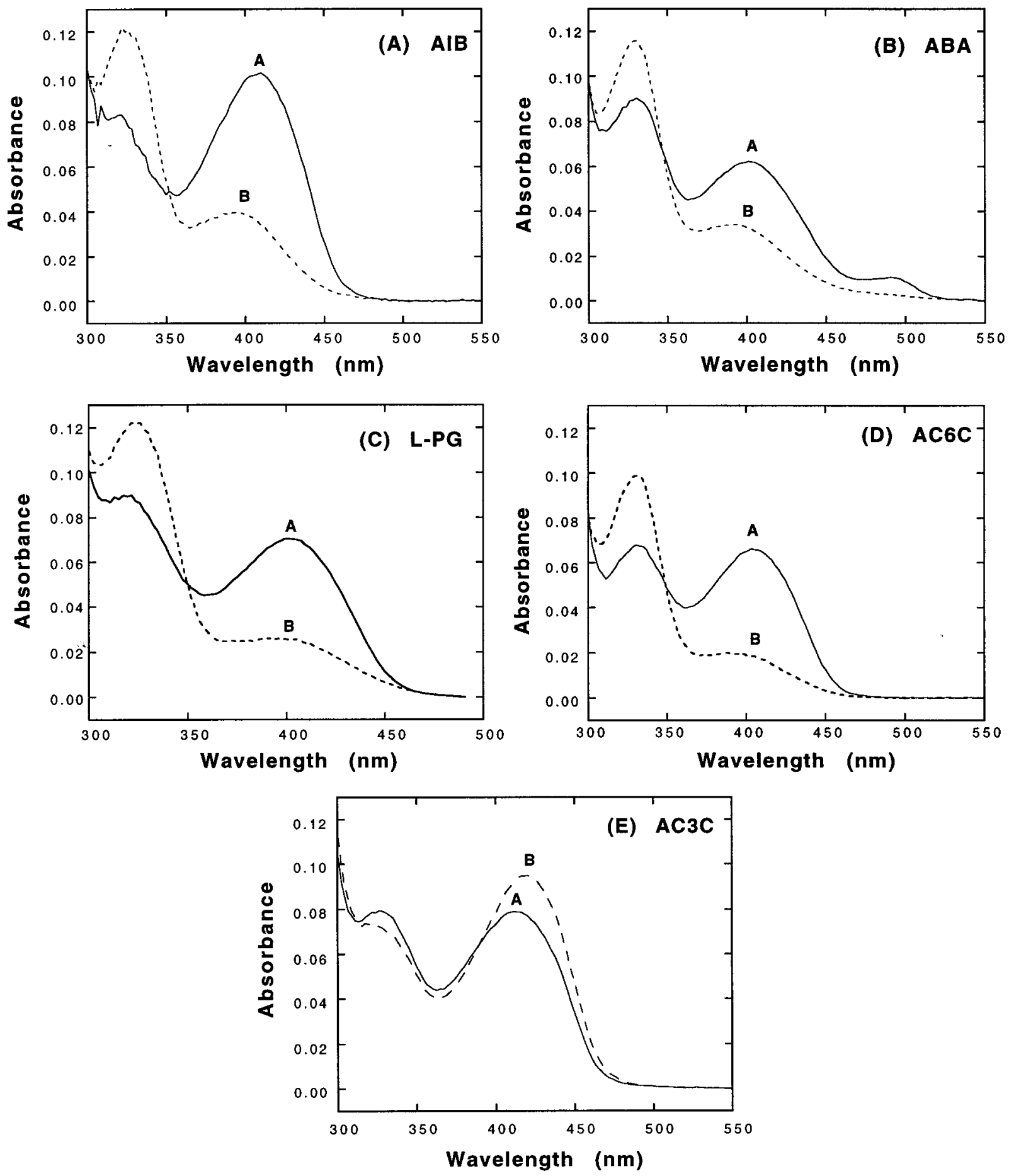

FIGURE 1: Absorbance spectra of the two species in the pre-steady-state reactions of substrates reacting in a single kinetic phase with the fast-reacting enzyme form. The decrease in absorbance at $\sim 400 \mathrm{~nm}$ of the initial species (A, solid line) and the increase in absorbance at $\sim 330 \mathrm{~nm}$ observed in the final species (B, dotted line) correspond to the conversion of DGD-PLP to DGD-PMP, except in the case of AC3C, which only reacts are far as the external aldimine: (A) AIB decarboxylation, (B) L-aminobutyrate transamination, (C) L-phenylglycine decarboxylation, (D) AC6C decarboxylation, and (E) AC3C external aldimine formation. The $\sim 500 \mathrm{~nm}$ absorbance in the spectrum of species A with L-aminobyturate is characteristic of a quinonoid intermediate. External aldimine formation followed by deprotonation to give the quinonoid thus occurs in the deadtime of the stopped-flow instrument $(1.6 \mathrm{~ms})$.

Both L-phenylglycine and AC6C show monophasic presteady-state kinetics. The values of $k_{\mathrm{obs}}$ for these substrates obtained with the stopped-flow spectrophotometer (Table 1) are close to the values measured in steady-state assays under identical conditions (3). The absorption spectra for Lphenylglyine and AC6C are shown in Figure 1C and D, respectively. If data collection were extended to very long times (which was not possible with the instrument used), one would expect to see the reaction of the slow form of the enzyme with these substrates also.

No decarboxylation reaction between DGD-PLP and AC3C was detected in steady-state experiments (3), yet it is a competitive inhibitor with $K_{\mathrm{i}}=1.4 \mathrm{mM}$ under the present conditions. AC $3 \mathrm{C}$ forms an external aldimine with DGD- 
Table 1: Decarboxylation and Transamination Rate Constants from Pre-Steady-State Kinetics ${ }^{a}$

\begin{tabular}{|c|c|c|c|c|}
\hline \multicolumn{2}{|c|}{ monophasic kinetics } & \multicolumn{3}{|c|}{ biphasic kinetics } \\
\hline substrate & $k\left(\mathrm{~s}^{-1}\right)$ & substrate & $k_{\text {fast }}\left(\mathrm{s}^{-1}\right)$ & $k_{\text {slow }}\left(\mathrm{s}^{-1}\right)$ \\
\hline AIB & $25(1)$ & MAM & $2.8(0.2)$ & $5.1(0.4) \times 10^{-3}$ \\
\hline L-Ala & $22(1)$ & AC5C & $7.5(1.0)$ & $0.54(0.07)$ \\
\hline L-aminobutyrate ${ }^{b}$ & $3.4(0.2)$ & isopropylamine & $2.6(0.5) \times 10^{-2}$ & $8.8(0.9) \times 10^{-3}$ \\
\hline L-phenylglycine & $0.11(0.01)$ & glycine $^{c}$ & $2.8(1.0) \times 10^{-2}$ & $7.6(2.0) \times 10^{-3}$ \\
\hline AC6C & $1.2(0.3)$ & & & \\
\hline $\mathrm{AC} 3 \mathrm{C}^{d}$ & $0.46(0.03)$ & & & \\
\hline
\end{tabular}

${ }^{a}$ Conditions: $30 \mathrm{mM}$ TEA-HCl, $\mathrm{pH} 8.0,15-21 \mu \mathrm{M}$ DGD, $0.1 \mathrm{M} \mathrm{KCl}$, varying concentrations of substrate, $25^{\circ} \mathrm{C}$. The rate constants reported here are the maximal values for AIB, L-Ala, L-aminobutyrate, AC3C, MAM, AC5C, and glycine. For the other substrates, the concentrations at which the rate constants were measured were as follows: isopropylamine, $230 \mathrm{mM}\left(K_{\text {app }}=94 \mathrm{mM}\right)$; L-phenylglycine, $10 \mathrm{mM}\left(K_{\text {app }}=8 \mathrm{mM}\right)$; AC6C, $80 \mathrm{mM}\left(K_{\text {app }}=54 \mathrm{mM}\right)$. Errors are given in parentheses. ${ }^{b}$ The value reported here is measured by the absorbance changes over the range of 300-550 nm. The rate constants measured by the LDH coupled assay and fluorescence data are $\sim 7 \mathrm{~s}^{-1}$. ${ }^{c}$ Steady-state kinetics shows that glycine undergoes transamination and decarboxylation at extremely slow rates (3). The half-time for these reactions is $\sim 1900 \mathrm{~s}$, while data collection proceeded for $1000 \mathrm{~s}$ here. ${ }^{d} \mathrm{AC} 3 \mathrm{C}$ reacts only as far as external aldimine formation.

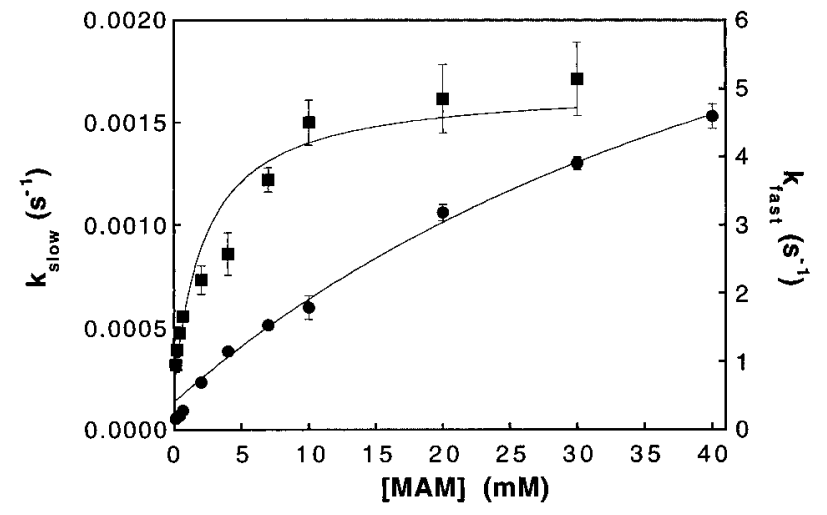

FIGURE 2: Observed rate constants as a function of concentration for MAM decarboxylation. Rate constants for both $k_{\text {fast }}$ (circles) and $k_{\text {slow }}$ (squares) exhibit hyperbolic dependence on substrate concentration. The solid lines are the best fits to eqs 2 and 3 .

PLP as evidenced by absorbance (Figure 1E) and circular dichroism (see below) changes. The rate constant at saturating $\mathrm{AC} 3 \mathrm{C}\left(20 \mathrm{mM}, 14 \times K_{\mathrm{i}}\right)$ is $0.46 \pm 0.03 \mathrm{~s}^{-1}$.

Biphasic Pre-Steady-State Kinetics. The reaction of MAM with the fast form of DGD-PLP clearly displays biphasic kinetics. The two processes are well separated kinetically. The rate constants for both show saturation behavior (Figure 2 ). The limiting value for the fast phase is $\sim 1000$-fold larger than that for the slow, which is approximately equal to the steady-state $k_{\text {cat }}$ value (3). The spectral data do not show an isosbestic point. The spectra obtained from global analysis are shown in Figure 3A.

The minimal kinetic mechanism for the reaction of the fast form of DGD-PLP with MAM is given in Scheme 1. Assuming the first step is at equilibrium (i.e., $K_{\mathrm{S}}=k_{-1} / k_{1}$ ), the apparent rate constants for the fast phase, $k_{\text {fast }}$, and the slow phase, $k_{\text {slow }}$, can be expressed in terms of the rate constants in Scheme 1, eqs 2 and 3. The derivations of eqs

$$
\begin{gathered}
k_{\text {fast }}=\frac{k_{2} \mathrm{~S}}{K_{\mathrm{S}}+\mathrm{S}}+k_{-2} \\
k_{\text {slow }}=\frac{k_{4} \mathrm{~S}}{K_{\mathrm{S}} \frac{k_{-2}}{k_{2}}+\left(1+\frac{k_{-2}}{k_{2}}\right) \mathrm{S}}+k_{\mathrm{PLP}}
\end{gathered}
$$

2 and 3 appear in the Appendix. Free PLP undergoes slow decomposition during data acquisition due to the intense
Scheme $1^{a}$

$$
E+S \underset{k_{-1}}{\stackrel{k_{1}}{\rightleftharpoons}} E S \underset{k_{2}}{\stackrel{k_{2}}{\rightleftharpoons}} E M \stackrel{k_{4}}{k_{3}} E+P_{1}
$$

${ }^{a}$ E represents DGD-PLP, ES the (noncovalent) Michaelis complex, EM the external aldimine, and $\mathrm{E}^{\prime}$ the PMP enzyme form. $\mathrm{P}_{1}$ and $\mathrm{P}_{2}$ are the products of nonoxidative (alanine) and oxidative (pyruvate) decarboxylation, respectively. The ratio of nonoxidative to oxidative decarboxylation $\left(k_{3} / k_{4}\right)$ is $1.3: 1(3)$.

Scheme 2

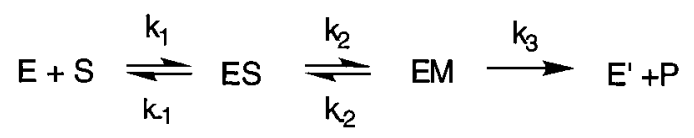

white light used with the diode array detector. This is accounted for by the $k_{\text {PLP }}$ term in eq 3 for the slow phase.

The observed values of $k_{\text {fast }}$ and $k_{\text {slow }}$ vs MAM concentration were fitted to the above equations. The values of the constants obtained from the fitting are as follows: $K_{\mathrm{S}}=61$ $\pm 21 \mathrm{mM}, k_{2}=3.5 \pm 0.8 \mathrm{~s}^{-1}, k_{-2}=0.11 \pm 0.01 \mathrm{~s}^{-1}, k_{3}=$ $(6.0 \pm 0.6) \times 10^{-3} \mathrm{~s}^{-1}, k_{4}=(4.6 \pm 0.4) \times 10^{-3} \mathrm{~s}^{-1}$, and $k_{\mathrm{PLP}}=(0.65 \pm 0.24) \times 10^{-3} \mathrm{~s}^{-1}$. Figure 2 shows the fit to the data.

The rate constants observed in the reaction of AC5C with DGD-PLP show similar behavior (Figure 4). The absorption spectra obtained from global analysis with this substrate are shown in Figure 3B. An insignificant amount of nonoxidative decarboxylation is observed with AC5C (3). The reaction is thus described by the mechanism given in Scheme 2. The following relations for the fast and slow rate constants are derived in the Appendix, based in Scheme 2.

$$
\begin{gathered}
k_{\text {fast }}+k_{\text {slow }}=\frac{\mathrm{S}}{K_{\mathrm{S}}+\mathrm{S}} k_{2}+k_{-2}+k_{3} \\
k_{\text {fast }} k_{\text {slow }}=\frac{\mathrm{S}}{K_{\mathrm{S}}+\mathrm{S}} k_{2} k_{3}
\end{gathered}
$$

Fitting the substrate concentration dependence of the observed $k_{\text {fast }}$ and $k_{\text {slow }}$ to eqs 4 and 5 yields $K_{\mathrm{S}}=26 \pm 14$ $\mathrm{mM}, k_{2}=9.0 \pm 1.5 \mathrm{~s}^{-1}, k_{-2}=0.55 \pm 0.40 \mathrm{~s}^{-1}$, and $k_{3}=$ $0.48 \pm 0.15 \mathrm{~s}^{-1}$. The fitting results are plotted in Figure 4 (solid lines) and generally account well for the data.

Absorption spectra obtained from global analysis of the reaction of DGD-PLP with isopropylamine are shown in 

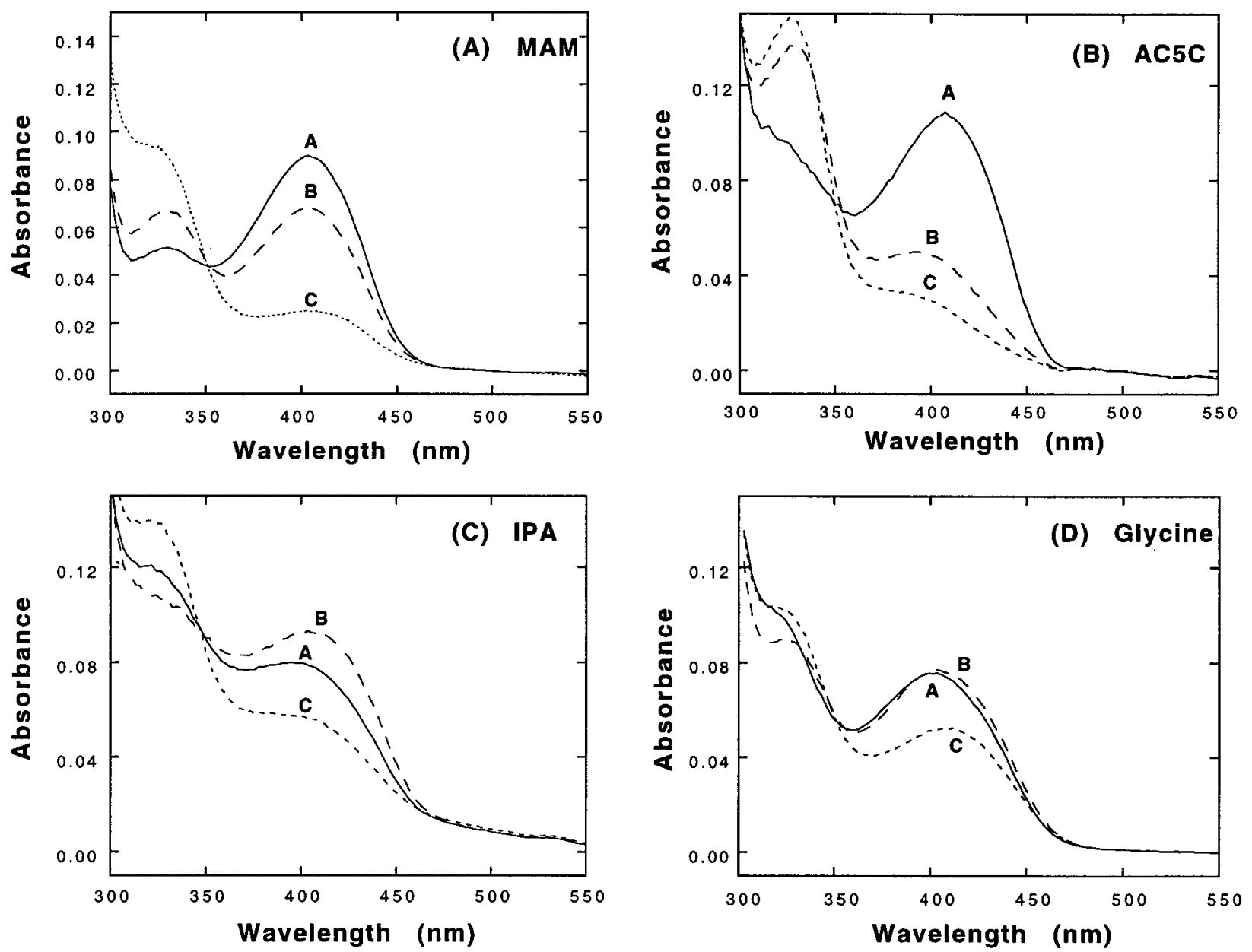

FIGURE 3: Absorbance spectra for the three kinetically distinguishable species in the reactions of substrates exhibiting biphasic pre-steadystate kinetics: initial species (A, solid line), intermediate (B, dashed line), and final species (C, dotted line). (A) MAM, (B) AC5C, (C) isopropylamine, and (D) glycine. The first kinetic phase for each substrate is proposed to be external aldimine formation. The second kinetic phase is proposed to be decarboxylation (MAM, AC5C) or transamination (isopropylamine, glycine).

Figure $3 \mathrm{C}$. The rate constants for the fast and slow phases at a concentration of $0.23 \mathrm{M}\left(3 \times K_{\mathrm{m}}\right)$ are $k_{\text {fast }}=(2.6 \pm$ $0.5) \times 10^{-2} \mathrm{~s}^{-1}$ and $k_{\text {slow }}=(8.8 \pm 0.9) \times 10^{-3} \mathrm{~s}^{-1}$. The fast phase occurs with an increase in long-wavelength absorbance and a slight red shift (Figure 3C), indicative of external aldimine formation. The slow-phase spectral changes are consistent with conversion of DGD-PLP to DGD-PMP via transamination.

The reaction of glycine with DGD-PLP is slow with a calculated half-time of $\sim 1900 \mathrm{~s}$ (3). Absorption spectra obtained from global analysis of the reaction of DGD-PLP with glycine over $1000 \mathrm{~s}$ are shown in Figure 3D. The estimated rate constants for the fast and slow phases at a concentration of $1.4 \mathrm{M}\left(6 \times K_{\mathrm{m}}\right)$ are $k_{\text {fast }}=(3.8 \pm 0.4) \times$ $10^{-2} \mathrm{~s}^{-1}$ and $k_{\text {slow }}=(6.4 \pm 0.4) \times 10^{-3} \mathrm{~s}^{-1}$.

LDH Coupling and Fluorescence Analysis of the $L^{-}$ Aminobutyrate Reaction. The transamination reaction of L-aminobutyrate with DGD-PLP was coupled in the stopped flow under pre-steady-state conditions to a large excess of LDH activity. Two kinetic processes were observed with rate constants of $6.9 \pm 1.6$ and $0.024 \pm 0.005 \mathrm{~s}^{-1}$. The same transamination reaction was monitored by the increase in coenzyme fluorescence (ex $330 \mathrm{~nm}$, em >360 nm) under identical conditions. The observed rate constants for the two kinetic phases are $8.0 \pm 0.4$ and $0.021 \pm 0.002 \mathrm{~s}^{-1}$.

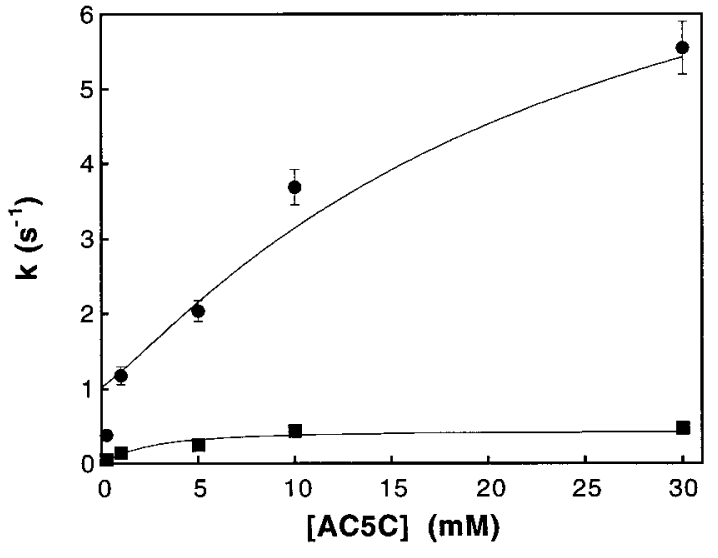

FIGURE 4: Observed rate constants as a function of concentration for the AC5C decarboxylation reaction. Rate constants for both $k_{\text {fast }}$ (circles) and $k_{\text {slow }}$ (squares) exhibit hyperbolic dependence on substrate concentration. The solid lines are the best fits to eqs 4 and 5.

Circular Dichroism Spectra and Kinetics. The CD spectra of DGD-PLP before and after the oxidative decarboxylation half-reaction with AIB are shown in Figure 5. PLP in the active site of the unliganded enzyme shows positive Cotton effects for both the $\sim 400$ - and $\sim 330$-nm absorption bands. That for the former is substantially larger. On decarboxy- 


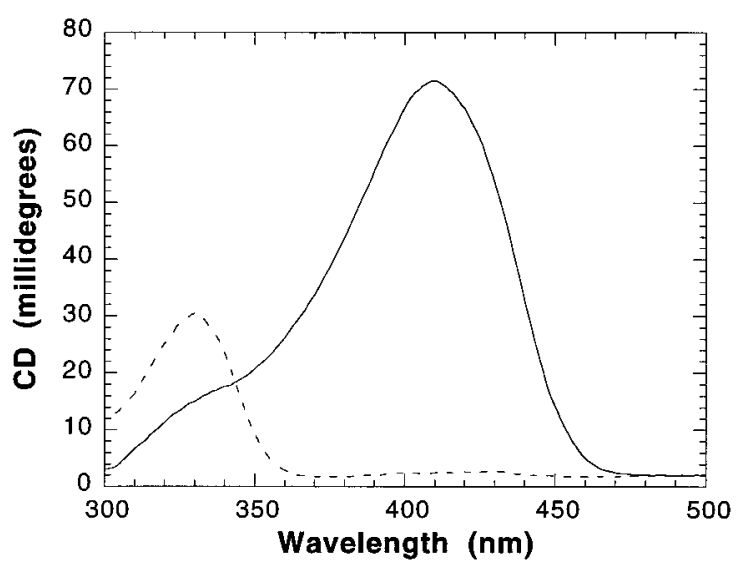

FIGURE 5: CD spectra of DGD-PLP before (solid line) and after (dotted line) the decarboxylation reaction with AIB. DGD-PLP exhibits a major absorbance band at $\sim 400 \mathrm{~nm}$ and a minor band at $\sim 330 \mathrm{~nm}$. After decarboxylation, the $\sim 400$-nm signal disappears and the 330-nm signal increases due to the conversion of DGDPLP to DGD-PMP.

lation, the $\sim 400-\mathrm{nm}$ CD decreases and the $\sim 330-\mathrm{nm}$ CD increases due to PMP formation.

The CD changes observed when DGD-PLP is mixed with pyruvate, lactate, AC3C, or MAM are shown in Figure 6. The time courses for the CD maxima of the final species are plotted in Figure 7.

There is slow increase in $\sim 400$-nm CD on mixing DGDPLP with pyruvate $\left(K_{\text {is }}=10 \mathrm{mM}\right)$ or lactate $\left(K_{\text {is }}=0.13\right.$ $\mathrm{M})$. These data are well accommodated by a singleexponential model. The observed rate constants are $\sim 5 \times$ $10^{-3} \mathrm{~s}^{-1}$ for pyruvate and $(2.0 \pm 0.1) \times 10^{-3} \mathrm{~s}^{-1}$ for lactate.

The $\mathrm{CD}$ spectral changes shown in Figure $6 \mathrm{C}$ are observed in the reaction of AC3C with DGD-PLP. The $\sim 400-\mathrm{nm}$ peak rapidly shifts to $\sim 420 \mathrm{~nm}$ within the $\sim 2 \min$ it takes to mix and begin data collection. A slower increase in longwavelength $\mathrm{CD}$ occurs over $\sim 25 \mathrm{~min}$, as with pyruvate and lactate. The rate constant for the slow process at $420 \mathrm{~nm}$ is $(1.4 \pm 0.1) \times 10^{-3} \mathrm{~s}^{-1}$ in the presence of $20 \mathrm{mM} \mathrm{AC} 3 \mathrm{C}$.

The reaction of MAM with DGD-PLP shows a rapid decrease in $\sim 400$-nm CD which is accompanied by a small red shift in the absorption maximum (Figure 6D). This fast process is complete within the $\sim 2 \min$ it takes to mix and begin data collection. A slower process occurs over $\sim 25$ min with $k_{\text {obs }}=(2.0 \pm 0.2) \times 10^{-3} \mathrm{~s}^{-1}$ at $20 \mathrm{mM}$ MAM. This agrees well with the value of $k_{\text {slow }}$ expected based on the absorbance data (Figure 3A) and is very similar to the steady-state $k_{\text {cat }}$ value (3).

Solvent Isotope Effects. The solvent isotope effects for the MAM reaction are ${ }^{\mathrm{D}_{2}} \mathrm{o}_{\text {fast }}=1.8 \pm 0.2$, and ${ }^{\mathrm{D}_{2} \mathrm{O}} k_{\text {slow }}=$ $1.2 \pm 0.1$. These solvent isotope effects are obtained at high concentration of MAM, under which condition $k_{\text {fast }} \approx k_{2}$, and $k_{\text {slow }} \approx k_{4}$. Thus, the observed solvent isotope effects on $k_{\text {fast }}$ and $k_{\text {slow }}$ approximate the intrinsic effects on external aldimine formation and the rate-limiting step for $\mathrm{CO}_{2}$ loss from the external aldimine, respectively.

An inverse solvent isotope effect for external aldimine formation with $\mathrm{AC} 3 \mathrm{C}$ is observed $\left(\mathrm{D}_{2} \mathrm{O} k=0.48 \pm 0.02\right)$. Smaller inverse isotope effects are observed for both rate constants in the $\mathrm{AC} 5 \mathrm{C}$ reaction $\left(\mathrm{D}_{2} \mathrm{O}_{\text {fast }}=0.78 \pm 0.02\right.$, $\left.\mathrm{D}_{2}{ }^{\circ} k_{\text {slow }}=0.74 \pm 0.09\right)$. The solvent isotope effect for AC6C is normal $\left(\mathrm{D}_{2} \mathrm{O} k=1.5 \pm 0.1\right)$.

\section{DISCUSSION}

Origin and Nature of Multiple Kinetic Processes. To avoid confusion, it is important to define clearly the two sources of biphasic character that have been observed. One source is the coexistence of two kinetically distinguishable, slowly interconverting enzyme forms (X. Zhou and M. D. Toney, submitted). The other is the mechanistic complexity of reactions of particular substrates with the fast-reacting form of DGD-PLP.

The slow phases in the AIB decarboxylation half-reaction and in the L-alanine and L-aminobutyrate transamination halfreactions are due to the independent reaction of a coexisting, slowly reacting enzyme form. This is most clearly demonstrated here by the observation of two kinetic processes when the transamination of L-aminobutyrate is coupled in the presteady state to the $\mathrm{LDH}$-catalyzed oxidation of NADH. This shows unequivocally that pyruvate is released into solution in two kinetic processes, the parallel reactions of fast and slow enzyme forms. The similarity of the rate constants observed in this experiment and in those monitored by absorbance and fluorescence corroborates this conclusion.

The presence of single, tight isosbestic points in data covering both kinetic processes for reactions of AIB, $\mathrm{L}$-alanine, and L-aminobutyrate is independent evidence that parallel reactions of two enzyme forms are being observed. Additionally, the rate constant for the slow phase in the AIB half-reaction is slower than the steady-state $k_{\text {cat }}$ value under identical conditions, ruling it out as part of the catalytic cycle of a homogeneous enzyme population.

Importantly, AIB, L-alanine, and L-aminobutyrate react in a single kinetic process with the fast-reacting enzyme form and are thus dubbed "monophasic" in the present context. The reactions of the slower enzyme form are not of great interest here since $>95 \%$ of catalysis in steady-state reactions under the present conditions is due to the fast-reacting enzyme form. Presumably, biphasicity due to two enzyme forms would also be observed in the reactions of Lphenylglycine and AC6C if data collection had been extended to much longer times (which was not possible due to instrumental limitations).

The second, more important source of biphasic character for the present analysis is the kinetic complexity of the reactions of MAM, AC5C, glycine, and isopropylamine with the fast-reacting enzyme form. Again, presumably if data collection for these substrates were continued to much longer times, the reactions of two enzyme forms would be observed.

Substrates Exhibiting Monophasic Kinetics with the FastReacting Enzyme Form. AIB reacts via a currently undefined rate-limiting step. Rapid formation of the external aldimine intermediate is suggested by the spectral shift from the free enzyme observed in the spectrum of the first species obtained from global analysis. Thus, $\mathrm{CO}_{2}$ loss is likely to be rate limiting. Rate constants for external aldimine formation in other well-studied PLP-dependent enzymes cover a broad range. In aspartate aminotransferase, external aldimine formation is a rapid equilibrium process (6), while with glutamate decarboxylase, for example, it is partially rate determining for the catalytic cycle (7).

With L-alanine and L-aminobutyrate, the spectrum from global analysis for the initial species has absorption near 500 $\mathrm{nm}$ characteristic of carbanionic quinonoid intermediates (5). 

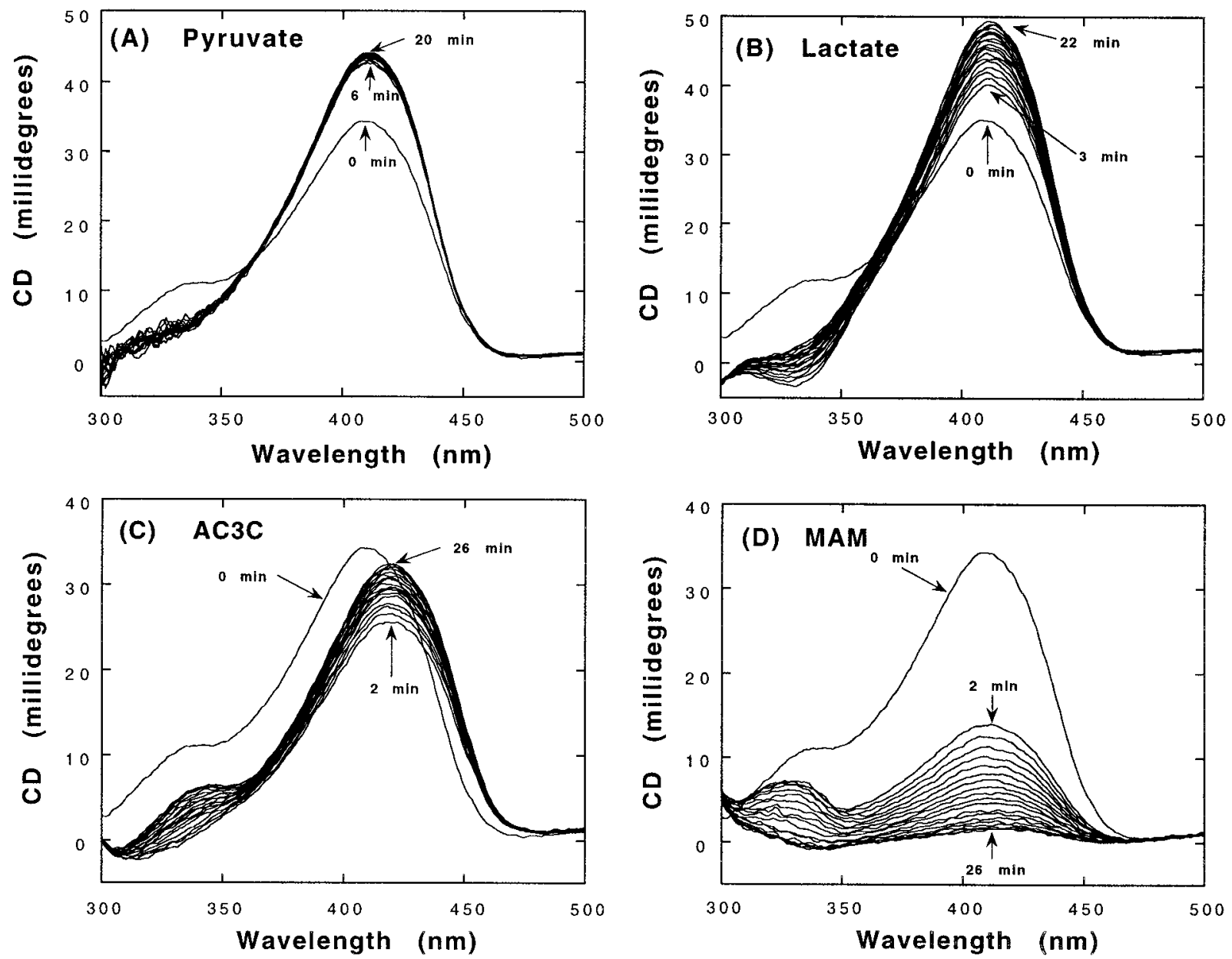

FIGURE 6: CD spectra of DGD-PLP after mixing with various substrate analogues: (A) pyruvate, (B) lactate, (C) AC3C, and (D) MAM. Pyruvate and lactate are competitive inhibitors. Their binding to DGD-PLP causes a slow increase in the $\sim 400-\mathrm{nm}$ CD. The reaction of AC3C with DGD-PLP rapidly shifts the long-wavelength CD band from $\sim 400$ to $\sim 420 \mathrm{~nm}$, followed by a slow increase in the 420 -nm signal. The reaction of MAM with DGD-PLP shows a rapid decrease in the $\sim 400$-nm signal followed by a slower decrease.

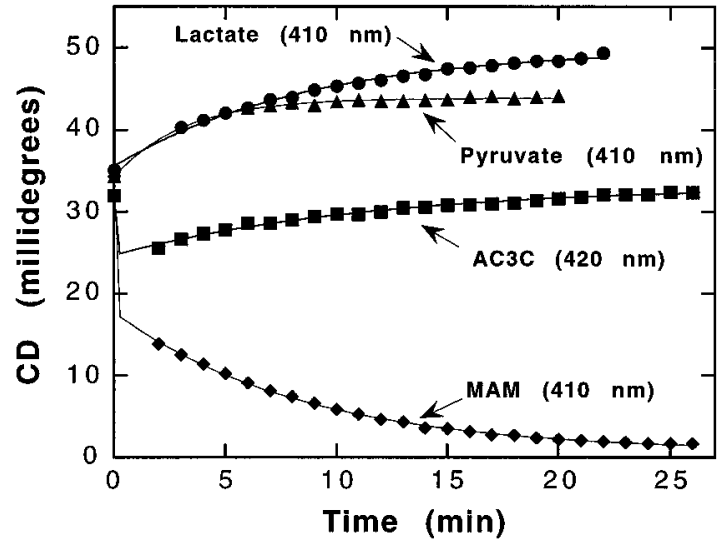

FIGURE 7: Time courses for CD signals at the wavelengths indicated for the reactions of pyruvate, lactate, $\mathrm{AC} 3 \mathrm{C}$, and MAM. The solid lines are best fits to a single-exponential model for pyruvate and lactate and to a double-exponential model for AC3C and MAM.

Two processes, external aldimine formation followed by loss of a proton from $\mathrm{C}^{\alpha}$ (Scheme 3 ), must occur in the dead time of the stopped flow for this absorption to be present in the spectrum of the initial species. These data alone point to a step following quinonoid formation as rate determining in transamination of these substrates.

The reactions of L-phenylglycine and $\mathrm{AC} 6 \mathrm{C}$ also exhibit monophasic half-reaction kinetics. The spectra obtained
Scheme 3

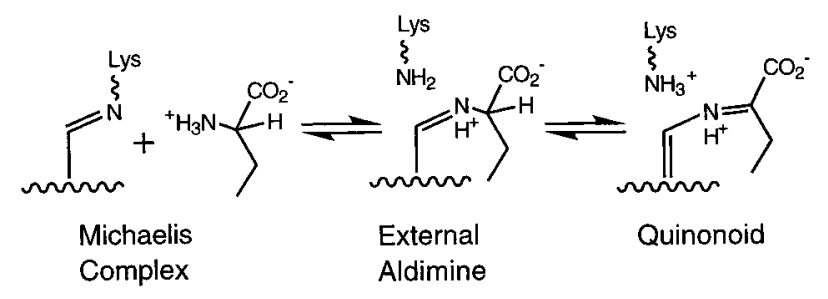

from global analysis do not show a red-shifted longwavelength peak for the initial species and thus external aldimine formation is likely to be rate limiting. The normal solvent isotope effect of 1.5 for AC6C is similar to that of 1.8 for external aldimine formation with MAM, consistent with this proposition.

Substrates Exhibiting Biphasic Kinetics with the FastReacting Enzyme Form. MAM, AC5C, isopropylamine, and glycine exhibit biphasic kinetics with the fast-reacting form of DGD. Unlike in the reaction of AIB, where a single isosbestic point is observed for both kinetic processes, the reactions of these substrates do not show a single isosbestic point in data covering the two kinetic processes. This suggests that these processes are mechanistically dissimilar.

The fast kinetic processes in the reactions of MAM and AC5C with DGD-PLP exhibit decreases in the long ( $\sim 400$ $\mathrm{nm})$ and increases in the short $(\sim 330 \mathrm{~nm})$ wavelength 
absorption peaks, while the isopropylamine reaction gives opposite changes. The reaction of the competitive inhibitor AC3C with DGD-PLP, which proceeds only as far as the external aldimine, exhibits spectral changes similar to those occurring in the fast phase of the isopropylamine reaction. The disparate spectral changes notwithstanding, the most attractive choice for the identity of the first kinetic process for MAM, AC5C, and isopropylamine alike is external aldimine formation. This assignment is supported by the observed rapid decrease and red shift in long-wavelength $\mathrm{CD}$ observed in the reactions of both $\mathrm{AC} 3 \mathrm{C}$ and MAM (Figure $6 \mathrm{C}$ and D, respectively) within the $\sim 2$-min mixing time before data collection was begun.

The differences in spectral changes observed for the fast processes with these substrates is readily explained by the dependence of PLP aldimine tautomer equilibria on solvent polarity (8). That is, nonpolar solvents favor the enolimine tautomer, which absorbs at $\sim 330 \mathrm{~nm}$, while polar solvents favor the ketoenamine tautomer, which absorbs at $\sim 400 \mathrm{~nm}$. The differences in active site electrostatic microenvironments caused by the binding of the various substrates may lead to variation in the observed tautomer equilibrium constant, accounting for the observation of both increases and decreases in the 330/400 $\mathrm{nm}$ ratios.

Enigmatically Slow Decarboxylation of MAM. MAM is decarboxylated very much more slowly by DGD than expected. The data of Thanassi (9) and Kalyankar and Snell (10) allow one to calculate that MAM is decarboxylated $\sim 10^{5}$-fold faster than is AIB by pyridoxal in solution. It was expected that a substantial part of this difference in reactivity would be observed in the enzymatic reaction with DGD. Contrary to expectation, MAM is decarboxylated $\sim 10^{4}$-fold slower by DGD than is AIB. This corresponds to a $\sim 10^{9}$-fold difference in expected vs observed reactivity. This is remarkable since the relative solution reactivities of substrates are generally preserved in enzyme active sites, as demonstrated, for example, by linear free energy relationships (11). Enzymologists have often invoked conformational changes as cloaks for such enigmas. The following explanation for the poor reactivity of MAM resorts to the same, but not without supporting experimental evidence.

It was established above that the reaction of MAM occurs via relatively fast external aldimine formation followed by an undefined rate-determining step. The general chemical mechanism of PLP catalysis (12) does not require an intermediate between the external aldimine and quinonoid intermediates. $\mathrm{CO}_{2}$ loss from MAM is expected to be rapid from an aldimine that enjoys the catalytic advantages that the AIB aldimine does. Thus, the most likely causes of the slow decarboxylation are the following: (1) an unfavorable proton-transfer rate or equilibrium, (2) an unfavorable Michaelis complex/external aldimine equilibrium, (3) catalytically disadvantageous interactions between active site residues and MAM (i.e., substantially different interactions from those occurring with AIB), and (4) a slow requisite conformational change.

The first of these possibilities is ruled out by the observation that the slope of a plot of $\log k_{\text {slow }} \mathrm{vs} \mathrm{pH}$ is much less than 1 (data not shown). The second is ruled out as follows. The fitting of eqs 2 and 3 to the MAM data and eqs 4 and 5 to the AC5C data allows the definition of $k_{2}$ and $k_{-2}$ in Schemes 1 and 2. The ratios of these are the values of the equilibrium constants for formation of the external aldimines from the Michaelis complexes. These are 32 and 16 in favor of the external aldimine for MAM and AC5C, respectively. This demonstrates that the poor reactivities of these substrates compared to AIB does not result from an unfavorable Michaelis complex/external aldimine equilibrium constant.

Unfortunately, X-ray structures of AIB and MAM analogues bound at the active site are not currently available. Thus, to address the third possibility for poor MAM reactivity (i.e., that the AIB and MAM aldimines make consequentially different interactions with active site residues), PM3 semiempirical quantum mechanical energy minimization calculations including active site residues in contact with the substrate and coenzyme $(\sim 190$ atoms total) were performed for both aldimines using the program Spartan. Here, the X-ray coordinates (13) of the active site region were extracted and the backbone and $\mathrm{C}^{\alpha}$ 's were removed, the latter being replaced by hydrogen atoms. The phosphate group was replaced by a methyl group, which was held fixed. The hydrogens corresponding to the $\mathrm{C}^{\alpha}$ 's, plus the $\mathrm{C}^{\beta}$ 's, were held fixed while all other atoms were free to move. Lys 272 was in its protonated form. Minimization was performed until the structures converged to within $0.03 \mathrm{kcal} / \mathrm{mol}$. The external aldimine structures obtained (Figure 8) differ very little, mainly in the geometry of the interaction made by Arg406 with the substrate carboxylate group. Transitionstate location calculations with these active site models (M. D. Toney, unpublished results) show a substantially lower activation barrier for MAM vs AIB. These results suggest that the difference in AIB and MAM reactivity is not due to differences in interactions made in structurally optimized complexes.

The CD data provide evidence in favor of the fourth possibility, a slow requisite conformational change. PLP is not intrinsically optically active, but is induced to be so by the asymmetric binding environment of the active site. Changes in the asymmetry of the binding site, as might occur in protein conformational changes, will give changes in the optical activity (i.e., CD) of bound PLP. The binding of pyruvate and lactate, competitive inhibitors of DGD-PLP that form only noncovalent complexes, is expected to be fast. Thus, the single-exponential changes in $\mathrm{CD}$ observed over $\sim 20$ min are best explained as a conformational change that occurs in the enzyme-ligand complex.

The reaction of $\mathrm{AC} 3 \mathrm{C}$ proceeds as far as the covalent external aldimine intermediate, whose formation is observed kinetically in the rapid coenzyme absorbance changes discussed above. A rapid CD change is observed within the $\sim 1$-min mixing time which is also attributed to external aldimine formation. A similar rapid change in $\mathrm{CD}$ with MAM in the mixing time is likewise attributable to external aldimine formation. The fast $\mathrm{CD}$ change with $\mathrm{AC} 3 \mathrm{C}$ is followed by a slow one that occurs over $\sim 20 \mathrm{~min}$, similar to the results with the noncovalently binding inhibitors pyruvate and lactate. At saturating $\mathrm{AC} 3 \mathrm{C}$ concentrations, $k_{\mathrm{obs}}=(1.4 \pm 0.1) \times 10^{-3} \mathrm{~s}^{-1}$ for the slow phase. This is very similar to the value of $(2.0 \pm 0.2) \times 10^{-3} \mathrm{~s}^{-1}$ obtained for the maximal value of $k_{\text {slow }}$ for the MAM reaction.

The quantitative agreement of $k_{\text {obs }}$ for the slow CD changes with these varied ligands strongly suggests that they can be ascribed to a common event, a protein conformational change. Such a slow conformational change is reminiscent 


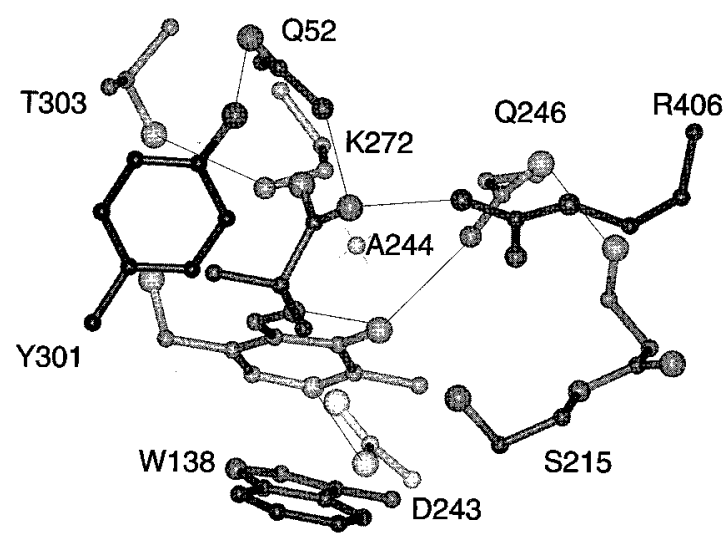

A

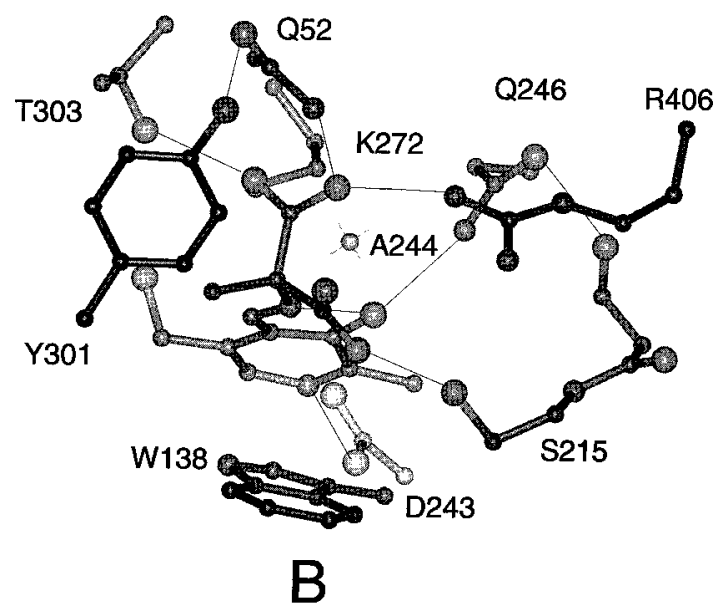

FIGURE 8: Model for the interaction of (A) AIB and (B) MAM with the DGD active site. The structures were obtained by energy minimization at the PM3 semiempirical quantum mechanical level. They show that the $\mathrm{C}^{\alpha}-\mathrm{CO}_{2}{ }^{-}$bond is in a catalytically efficient orientation for both substrates in structurally optimized complexes. Thin lines indicate hydrogen bonds.

of that observed by Onuffer and Kirsch (14) with the D222A mutant of aspartate aminotransferase.

Solvent Isotope Effects. MAM Schiff base formation occurs with a normal solvent isotope effect of 1.8 , similar to the solvent isotope effect of $\sim 2$ on model Schiff base formation (15). The solvent isotope effect on the slow phase of MAM is small or nonexistent, implying that any isotopesensitive steps (e.g. quinonoid protonation, Schiff base hydrolysis) are fast compared to the rate-determining step, suggested above to be a conformational change.

Schiff base formation with $\mathrm{AC} 3 \mathrm{C}$ is 2-fold faster in $\mathrm{D}_{2} \mathrm{O}$ than in $\mathrm{H}_{2} \mathrm{O}$ at high $\left(14 \times K_{\mathrm{i}}\right)$ concentrations. The reaction of AC3C with DGD-PLP includes Michaelis complex formation and Schiff base interchange. A very large inverse isotope effect on substrate binding would be required to offset the expected normal isotope effect on Schiff base interchange to give an overall inverse isotope effect. An unlikely alternative is that yet another conformational change is required prior to Schiff base interchange, which occurs with a large inverse isotope effect.

Sulfhydryl groups and metal-bound water molecules are known to give inverse solvent isotope effects (16). No cysteines are located in or near the active site of DGD, yet an alkali metal ion binding site is present (13). The identity of the alkali metal ion bound at this site controls the equilibrium distribution of fast and slow enzyme forms (X. Zhou and M. D. Toney, submitted). The potassium ion in the DGD form studied here has one water molecule as a ligand. This opens the possibility that Schiff base formation with $\mathrm{AC} 3 \mathrm{C}$ and $\mathrm{AC} 5 \mathrm{C}$ requires structural changes/ionizations at this metal site that the other substrates do not.

\section{ACKNOWLEDGMENT}

Critical reading of the manuscript and valuable comments provided by Professors R. L. Schowen and M. H. O'Leary are gratefully acknowledged.

\section{APPENDIX}

The minimal kinetic mechanism for the reaction of DGD with MAM is given by Scheme 1. Here, the formation of ES is assumed to be rapid and at equilibrium compared to the subsequent processes, i.e.:

$$
\frac{\mathrm{E} \cdot \mathrm{S}}{\mathrm{ES}}=\frac{k_{-1}}{k_{1}} \equiv K_{\mathrm{S}}
$$

Since $\mathrm{E}+\mathrm{ES}+\mathrm{EM}+\mathrm{E}^{\prime}=\mathrm{E}_{0}$, one obtains

$$
\mathrm{ES}=\frac{\mathrm{S}}{K_{\mathrm{S}}+\mathrm{S}}\left(\mathrm{E}_{\mathrm{o}}-\mathrm{EM}-\mathrm{E}^{\prime}\right)
$$

From Scheme 1

$$
\begin{gathered}
\frac{\mathrm{dEM}}{\mathrm{d} t}=k_{2} \mathrm{ES}-\left(k_{-2}+k_{3}+k_{4}\right) \mathrm{EM} \\
\mathrm{dE}^{\prime} / \mathrm{d} t=k_{4} \mathrm{EM}
\end{gathered}
$$

Substituting eq A2 into A3 for ES and taking the second derivative of the result one gets

$$
\begin{array}{r}
\frac{\mathrm{d}^{2} \mathrm{EM}}{\mathrm{d} t^{2}}+\left(\frac{\mathrm{S}}{K_{\mathrm{S}}+\mathrm{S}} k_{2}+k_{-2}+k_{3}+k_{4}\right) \frac{\mathrm{dEM}}{\mathrm{d} t}+ \\
k_{2} \frac{\mathrm{S}}{K_{\mathrm{S}}+\mathrm{S}} k_{4} \mathrm{EM}=0
\end{array}
$$

Substituting $\mathrm{e}^{-k t}$ for EM converts eq A5 into a quadratic in $k$. The apparent rate constants, $k_{\text {fast }}$ and $k_{\text {slow }}$, are obtained as the roots of this quadratic and are given in

$$
\begin{aligned}
& k_{\text {fast }, \text { slow }}=\frac{1}{2}\left[\frac{\mathrm{S}}{K_{\mathrm{S}}+\mathrm{S}} k_{2}+k_{-2}+k_{3}+k_{4} \pm\right. \\
& \left.\left(\left(\frac{\mathrm{S}}{K_{\mathrm{S}}+\mathrm{S}} k_{2}+k_{-2}+k_{3}+k_{4}\right)^{2}-4 k_{4} \frac{\mathrm{S}}{K_{\mathrm{S}}+\mathrm{S}} k_{2}\right)^{1 / 2}\right]
\end{aligned}
$$

As seen from the data in the Results section, $k_{\text {fast }} \gg k_{\text {slow }}$ and thus,

$$
\left(\frac{\mathrm{S}}{K_{\mathrm{S}}+\mathrm{S}} k_{2}+k_{-2}+k_{3}+k_{4}\right)^{2} \gg 4 k_{4} \frac{\mathrm{S}}{K_{\mathrm{S}}+\mathrm{S}} k_{2}
$$

since $k_{\text {fast }}$ will be the positive root and $k_{\text {slow }}$ the negative. Exploiting this, the apparent rate constants, $k_{\text {fast }}$ and $k_{\text {slow }}$, can be expressed in a simpler relationship to substrate concentration through a Taylor series expansion of the square root term in eq A6. The root is of the form $(1-x)^{1 / 2}$ with 
$x$ much smaller than 1 when both terms in the square root are divided by the first. The first two terms in the series are used for the expressions for $k_{\text {fast }}$ and $k_{\text {slow }}$, which are given in

$$
\begin{aligned}
& k_{\text {fast }}=\frac{k_{2} \mathrm{~S}}{K_{\mathrm{S}}+\mathrm{S}}- \\
& \frac{k_{2} k_{4} \mathrm{~S}}{K_{\mathrm{S}}\left(k_{-2}+k_{3}+k_{4}\right)+\left(k_{2}+k_{-2}+k_{3}+k_{4}\right) S}+k_{-2}+ \\
& k_{3}+k_{4} \text { (A7) } \\
& k_{\text {slow }}=\frac{k_{2} k_{4} \mathrm{~S}}{K_{\mathrm{S}}\left(k_{-2}+k_{3}+k_{4}\right)+\left(k_{2}+k_{-2}+k_{3}+k_{4}\right) \mathrm{S}}
\end{aligned}
$$

Assuming that $k_{2}, k_{-2} \gg k_{3}+k_{4}$, as is borne out by the fitting results, eqs $\mathrm{A} 7$ and $\mathrm{A} 8$ can be further approximated to eqs A9 and A10 (a background of slow PLP decomposi-

$$
\begin{gathered}
k_{\text {fast }}=\frac{k_{2} \mathrm{~S}}{K_{\mathrm{S}}+\mathrm{S}}+k_{-2} \\
k_{\text {slow }}=\frac{k_{4} \mathrm{~S}}{K_{\mathrm{S}}\left(k_{-2} / k_{2}\right)+\left(1+\left(k_{-2} / k_{2}\right)\right) \mathrm{S}}+k_{\mathrm{PLP}}
\end{gathered}
$$

tion $k_{\mathrm{plp}}$ is included in the calculation of $\left.k_{\mathrm{slow}}\right)$.

The ratio of nonoxidative to oxidative decarboxylation is 1.3 (3), thus $k_{3}=1.3 k_{4}$. The four unknown parameters $K_{\mathrm{S}}$, $k_{2}, k_{-2}$, and $k_{4}$ are obtained by fitting the hyperbolic dependence of the observed rate constants to eqs A9 and A10.

For AC5C, a similar kinetic mechanism (Scheme 2) holds except that the nonoxidative decarboxylation pathway is insignificant (3). The apparent rate constants are derived as described above, and given in eq A11: As seen in the

$$
\begin{aligned}
k_{\text {fast,slow }} & =\frac{1}{2}\left[\frac{\mathrm{S}}{K_{\mathrm{S}}+\mathrm{S}} k_{2}+k_{-2}+k_{3} \pm\right. \\
& \left.\left(\left(\frac{\mathrm{S}}{K_{\mathrm{S}}+\mathrm{S}} k_{2}+k_{-2}+k_{3}\right)^{2}-4 \mathrm{k}_{3} \frac{\mathrm{S}}{K_{\mathrm{S}}+\mathrm{S}} k_{2}\right)^{1 / 2}\right]
\end{aligned}
$$

pre-steady-state data, the two observed rate constants for
AC5C are of the same order of magnitude. Thus, a Taylor expansion cannot be applied to the square root in eq A11. Alternatively, the dependence of the observed rate constants on substrate concentration can be analyzed with the relationships given in eqs A12 and A13.

$$
\begin{gathered}
k_{\text {fast }}+k_{\text {slow }}=\frac{\mathrm{S}}{K_{\mathrm{S}}+\mathrm{S}} k_{2}+k_{-2}+k_{3} \\
k_{\text {fast }} k_{\text {slow }}=\frac{\mathrm{S}}{K_{\mathrm{S}}+\mathrm{S}} k_{2} k_{3}
\end{gathered}
$$

\section{REFERENCES}

1. Bailey, G. B., Chotamangsa, O., and Vuttivej, K. (1970) Biochemistry 9, 3243-3248.

2. Kallen, R. G., Korpela, T., Martell, A. E., Matsushima, Y., Metzler, C. M., Metzler, D. E., Morozov, Y. V., Ralston, I. M., Savin, F. A., Torchinsky, Y. M., and Ueno, H. (1984) in Transaminases (Christen, P., and Metzler, D. E., Eds.) John Wiley and Sons: New York.

3. Sun, S., Zabinski, R. F., and Toney, M. D. (1998) (previous paper in this issue).

4. Beechem, J. M. (1992) Methods Enzymol. 210, 37-53.

5. Metzler, C. M., Harris, A. G., and Metzler, D. E. (1988) Biochemistry 27, 4923-4933.

6. Kiick, D. M., and Cook, P. F. (1983) Biochemistry 22, 375382.

7. Albell, L. M., and O'Leary, M. H. (1988) Biochemistry 27, 3325-3330.

8. Shalteil, S., and Cortijo, M. (1970) Biochem. Biophys. Res. Commun. 41, 594-600.

9. Thanassi, J. W. (1972) Biochemistry 11, 2909-2914.

10. Kalyankar, G. D., and Snell, E. E. (1962) Biochemistry 1, 594600.

11. Kirsch, J. F. (1972) in Advances in Linear Free Energy Relationships (Chapman, N. B., and Shorter, J., Eds.) Chapter 8, Plenum, New York.

12. Metzler, D. E., Ikawa, M., and Snell, E. E. (1954) J. Am. Chem. Soc. 76, 648-652.

13. Toney, M. D., Hohenester, E., Keller, J. W., and Jansonius, J. (1995) J. Mol. Biol. 245, 151-179.

14. Onuffer, J. J., and Kirsch, J. F. (1994) Protein Eng. 7, 413424.

15. Fischer, H., DeCandis F. X., Ogden, S. D., and Jencks, W. P. (1980) J. Am. Chem. Soc. 76, 644-648.

16. Quinn, D. M., and Sutton, L. D. (1991) in Enzyme Mechanisms from Isotope Effects (Cook, P. F., Ed.) Chapter 3, CRC Press, Boca Raton, FL.

BI972056K 